\title{
Avaliação do Impacto da Carga de Saída em um Gerador de Energia Baseado no Efeito Piezoelétrico
}

\author{
Marcos Antonio Jeremias Coelho ${ }^{1}$ \\ Valner João Brusamarello ${ }^{1}$ \\ Jeferson Vieira Flores ${ }^{1}$
}

Programa de Pós-Graduação em Engenharia Elétrica - UFRGS, Porto Alegre, RS

\begin{abstract}
Resumo. Neste artigo será apresentado um estudo sobre um sistema de microgeração de energia a partir de vibração utilizando gerador piezoelétrico acoplado a uma viga em balanço. O sistema foi identificado observando suas características com as seguintes cargas: puramente resistiva e não linear (retificador onda completa). Por fim, é realizada a análise da potência gerada com estas cargas acopladas à saída.
\end{abstract}

Palavras-chave. Barras Engastadas, Carga Não Linear, Identificação de Sistema, Microgeração de Energia, Piezoelétricidade

\section{Introdução}

A microgeração de energia através de transdutores piezoelétricos baseia-se na conversão de energia mecânica em energia elétrica. Sua utilização é uma tendência crescente em aplicações como: nós sensores [3] e alimentação de circuitos integrados MEMS [5]. Em certas aplicações ocorre a integração de geração a partir da vibração mecânica com outras fontes como, por exemplo, solar [2].

Este estudo apresenta os resultados de um microgerador de energia elétrica a partir de vibração baseado no efeito piezoelétrico acoplado a uma viga de alumínio engastada em uma de suas extremidades. Realizou-se a montagem do experimento possibilitando a variação de frequência, amplitude do estímulo e tipos de cargas utilizadas (resistiva e não linear) a fim de validar os resultados analíticos encontrados. Para melhor compreensão do sistema foi realizada a identificação dos parâmetros individuais da viga, gerador piezoelétrico, cargas e condicionamento. Os diferentes tipo de cargas foram acopladas ao gerador verificando as potências geradas e sua influência no sistema, buscando assim a melhor condição para geração de energia elétrica.

\footnotetext{
${ }^{1}\{$ marcos.coelho, valner.brusamarello, jeferson.flores\}@ufrgs.br
} 


\section{Sistema de Microgeração de Energia}

Neste trabalho foi considerado o arranjo experimental da Figura 1, o qual consiste de uma lâmina flexível de alumínio engastada em uma das suas extremidades a uma base fixa. Dois transdutores piezoelétricos foram fixados em cada um dos lados da lâmina junto ao engaste. Desta forma, um transdutor age como atuador controlando a vibração da estrutura, e outro como gerador de energia provida desta vibração.
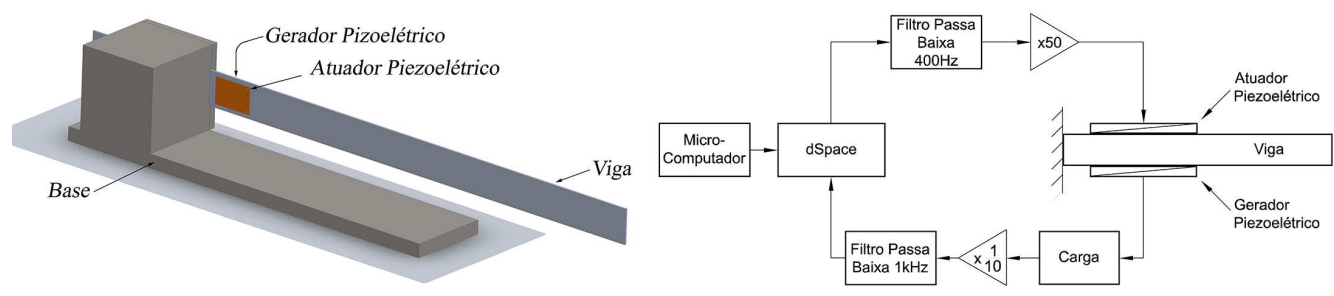

Figura 1: Estrutura Utilizada para o Gerador de Energia Baseado no Efeito Piezoelétrico.

As viga tem as seguintes características: Comprimento $(L) 0.71 \mathrm{~m}$, largura $(W) 0.05 m$, espessura $(h) 0.003 \mathrm{~m}$, constate de Young $(E) 7 \times 10^{10} \mathrm{~N} / \mathrm{m}^{2}$ e densidade de massa $(\rho)$ $2770 \mathrm{~kg} / \mathrm{m}^{3}$, onde o material da viga utilizada é alumínio. Os transdutores piezoelétricos: Comprimento $\left(L_{p}\right) 45.97 \mathrm{~mm}$, largura $\left(W_{p}\right) 33.27 \mathrm{~mm}$, espessura $\left(h_{p}\right) 0.864 \mathrm{~mm}$, constate de Young $\left(E_{p}\right) 6.7 \times 10^{10} \mathrm{~N} / \mathrm{m}^{2}$, constante de tensão $\left(g_{31}\right)-11.3 \times 10^{-3} \mathrm{Vm} / \mathrm{N}$, constante de carga $\left(d_{31}\right)-1.9 \times 10^{-10} \mathrm{~m} / \mathrm{V}$ e fator de acoplamento eletromecânico $\left(k_{31}\right) 0.36$. O atuador piezoelétrico é do tipo QP20W e o gerador piezoelétrico é do tipo V20W, ambos do fabricante Midé [8].

Na mesma figura é apresentado o diagrama completo do experimento. Também foi utilizada uma placa de processamento de sinais e controle em tempo real (dSpace), para a geração do sinal de excitação e aquisição de dados. O sinal é condicionado para ser aplicado ao atuador piezoelétrico acoplado a viga. Na face oposta ao atuador tem-se o gerador piezoelétrico onde os diferentes tipo de cargas são conectados. O condicionamento projetado consiste de um filtro passa-baixas para a frequência de corte em $400 \mathrm{~Hz}$, com um circuito de $1^{\mathrm{a}}$ ordem passivo responsável pela atenuação do ruído de quantização. Com a finalidade de gerar maior deformação na viga, o atuador foi alimentado em sua máxima tensão de $\pm 200 \mathrm{~V}$, conforme indicado pelo fabricante, necessitando assim de um amplificador com ganho $50 \mathrm{~V} / \mathrm{V}$ a partir de um sinal de referência. Para a aquisição do sinal gerado na carga utilizou-se um atenuador de 10x e um filtro passa-baixas anti-aliasing de segunda ordem sintonizado em $1 k H z$.

\subsection{Atuador Piezoelétrico Acoplado a Viga}

A partir de [8] obtém-se a seguinte função de transferência que relaciona a tensão aplicada no sensor e a deflexão da viga, assumindo apenas os $M$ primeiros modos de vibração mais significantes [4]:

$$
G_{v(s)}=k_{s} \frac{\prod_{i=1}^{M}\left(s^{2}+2 \hat{\zeta}_{i} \hat{\omega}_{i} s+\hat{\omega}_{i}^{2}\right)}{\prod_{i=1}^{M}\left(s^{2}+2 \zeta_{i} \omega_{i} s+\omega_{i}^{2}\right)}
$$


onde $\zeta_{i}$ e $\omega_{i}$ representam o coeficiente de amortecimento e frequência natural dos polos associados ao i-ésimo modo de vibração de $G_{v(s)}$. Os parâmetros $\hat{\zeta}_{i}$ e $\hat{\omega}_{i}$ denotam os parâmetros associados aos zeros do mesmo modo de vibração.

\subsection{Gerador Piezoelétrico}

Por apresentar um efeito dual, o gerador piezoelétrico tem o comportamento inverso ao atuador, sendo gerido pelas mesmas equações anteriormente apresentadas [6]. Porém, neste caso, o gerador irá converter a deformação em um rearranjo de cargas internas que ao ser acoplado a um circuito gerará uma corrente.

O circuito equivalente do gerador piezoelétrico é representado na Figura 2, onde $I_{p}$ é a corrente gerada, $C_{p}$ a capacitância interna, $R_{p}$ a resistência interna, $R_{d}$ a resistência de perdas, sendo $V_{s}$ a tensão gerada com circuito aberto e $V_{p}$ a tensão na saída do elemento piezoelétrico. O gerador piezoelétrico é posicionado para que ocorra a maior deformação no mesmo, sendo próximo a extremidade engastada. A tensão elétrica $V_{s}$ tem magnitude diretamente proporcional à deformação, tendo sua maior magnitude no primeiro modo de vibração, decaindo para os modos posteriores.
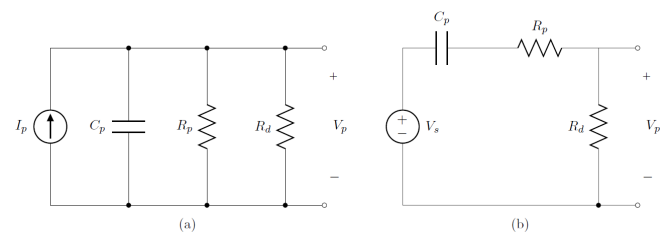

Figura 2: Circuito de Geração Piezoelétrico: (a) Fonte de Corrente; (b) Fonte de Tensão.

\subsection{Cargas}

O gerador piezoelétrico foi submetido a dois tipos distintos de cargas a fim de avaliar o seu comportamento: carga puramente resistiva, composta de um único resistor ligado a saída do elemento gerador, e a carga não linear, sendo um retificador de onda completa com resistência de carga e capacitor filtro. O modelo equivalente de cada uma das cargas é avaliado a seguir.

Para o caso de carga puramente resistiva foi aplicada a transformada de Laplace na equação das malhas do circuito com a carga resistiva conectada ao modelo de geração apresentado na Figura 2(b), onde a entrada é tensão $V_{s}(s)$ e saída a tensão na carga $V_{o}(s)$, obtendo-se:

$$
G_{l}(s)=\frac{K_{r} s}{s+\tau}, K_{r}=\frac{R_{d} \cdot R_{l} \cdot C_{p}}{R_{p} \cdot R_{l} \cdot C_{p}+R_{p} \cdot R_{d} \cdot C_{p}+R_{d} \cdot R_{l} \cdot C_{p}}, \tau=\frac{R_{l}+R_{d}}{R_{p} \cdot R_{l} \cdot C_{p}+R_{p} \cdot R_{d} \cdot C_{p}+R_{d} \cdot R_{l} \cdot C_{p}}
$$

onde $R_{l}$ a carga resistiva. Percebe-se que a função de transferência tem o valor do zero em frequências tendendo a zero, devido ao efeito do capacitor $C_{p}$ do modelo do gerador 
piezoelétrico que está conectado em série com o circuito. O valor do polo se dá em função do valor da carga $R_{l}$, já que os outros termos da equação tem valores fixos.

A análise da carga não linear (Circuito retificador) baseia-se no fato da corrente gerada pelo elemento piezoelétrico ter característica aproximadamente senoidal [9]. A inclusão do retificador de onda completa caracteriza um comportamento não linear na saída do sistema. A fim de aproximar o circuito retificador por uma carga linear, considera-se que a influência das harmônicas de ordem elevada no sinal de tensão não tem contribuição significativa na potência final gerada. Neste caso, apenas a componente fundamental $V_{e f}\left(j \omega_{o}\right)$ é considerada de tal forma que a impedância da carga vista pelo elemento gerador é razão entre esta tensão e a corrente $I_{p}\left(j \omega_{o}\right)[7]$.

\section{Identificação do Sistema}

O sinal de excitação utilizado na identificação da função de transferência do sistema é uma Sequência Pseudoaleatória Binária (PRBS) variando entre $\pm 4 V$ com período ajustável. A justificativa de utilização deste sinal é a semelhança com o ruído branco, sendo um sinal persistentemente excitante [1].

A geração do PRBS é realizada de forma digital, assim existe a presença do efeito da quantização, sendo necessária a utilização de um filtro passa-baixas definido como $G_{P B_{p}}(s)$. O amplificador é definido como $k_{a m p}$. O filtro passa-baixa ativo é definido como $G_{P B_{a}}(s)$. O conjunto do gerador piezoelétrico e carga tem sua função de transferência descrita em (2), sendo atribuído um e um zero em função do capacitor interno do modelo do piezoelétrico. No condicionamento de sinal estão os dois filtros passa baixas, sendo um de primeira ordem e um de segunda ordem, adicionando três polos ao sistema.

Assim o sistema a ser identificado é representado na Figura 3 com equação definida em (3), onde o modelo utilizado para a viga é descrito em (1) considerando apenas os quatro primeiros modos de vibração, totalizando quatros pares de polos e zeros complexos conjugados.

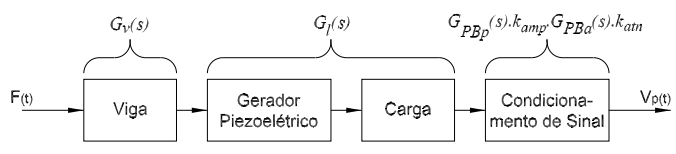

Figura 3: Representação do Sistema a ser Identificado

$$
G(s)=G_{P B_{p}}(s) k_{a m p} G_{v}(s) G_{l}(s) k_{a t n} G_{P B_{a}}(s)
$$

Na Figura 4(a) é apresentada a função de densidade espectral de potência do sinal de saída após o ensaio com o PRBS, na qual fica evidente as frequências naturais de vibração da viga da saída adquirida. Com auxilio da ferramenta System Identification Toolbox, do Matlab, a identificação do sistema foi realizada utilizando o método de Mínimos Quadrados Não linear. Na Figura 4(b) tem-se a comparação entre o diagrama de magnitude da resposta obtida no sistema teórico obtido a partir dos parâmetros da planta e da resposta obtida no procedimento de identificação. 

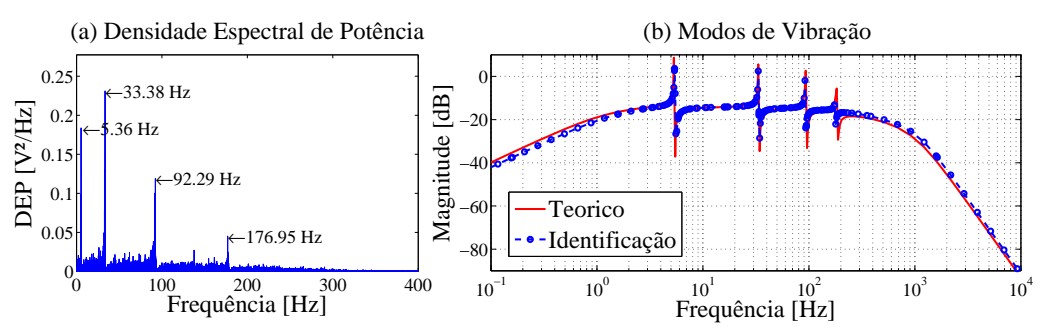

Figura 4: (a) Densidade Espectral de Potência com a Aplicação do PRBS e (b) Diagrama de Bode de Amplitude do Modelo Teórico e Identificado

Assim, os parâmetros relacionados aos modos de vibração da viga são apresentados na Tabela 1.

Tabela 1: Valores para Função de Transferência $G_{v}(s)$.

\begin{tabular}{c|c|c|c|c}
\hline Modo & $\hat{\omega}_{j}[\mathrm{~Hz}]$ & $\omega_{i}[\mathrm{~Hz}]$ & $\hat{\zeta}_{j}$ & $\zeta_{i}$ \\
\hline \hline $1^{\mathrm{o}}$ & 5.58 & 5.42 & 0.0046 & 0.0045 \\
\hline $2^{\mathrm{o}}$ & 34.10 & 33.38 & 0.004 & 0.004 \\
\hline $3^{\mathrm{o}}$ & 93.62 & 92.25 & 0.0039 & 0.0040 \\
\hline $4^{\mathrm{o}}$ & 179.18 & 177.89 & 0.0042 & 0.0039 \\
\hline
\end{tabular}

\section{Influência da Carga na Saída do Sistema}

Uma observação importante é a alteração de potência de acordo com o tipo de carga e frequência de excitação do sistema. O gerador piezoelétrico, que apresenta características capacitivas em sua impedância interna, influencia diretamente na potência entregue a carga. Em baixas frequências a impedância interna da fonte tem valor elevado em função do capacitor $C_{p}$, sendo este valor caindo exponencialmente com a elevação da frequência. Os valores de potência instantânea para uma carga puramente resistiva em função dos modos de vibração são apresentados na Tabela 2. Nota-se que, mesmo com a tensão elétrica no gerador sendo proporcional à deformação, a maior potência entregue a carga puramente resistiva não é obtida no primeiro modo de vibração para os resistores menores que $200 k \Omega$ por causa da impedância interna do gerador. Para os dois maiores resistores $(500 k \Omega$ e $1 M \Omega)$ a potência máxima se dá no primeiro modo de vibração, diminuindo nos modos mais elevados, já que nestas cargas a resistência tem valor muito superior a impedância da fonte. Para o primeiro modo de vibração (frequência de $5.42 \mathrm{~Hz}$ ) a maior potência se dá na resistência de $200 k \Omega$, próxima a resistência ótima teórica de $229,35 k \Omega$ para este modo. A potência máxima ocorre nas resistências de $50 K \Omega, 20 k \Omega$ e $10 k \Omega$ para o segundo, terceiro e quarto modo, respectivamente, comprovando a característica de diminuição da impedância da fonte com a elevação da frequência.

Para o caso da ligação da saída do micro gerador de energia no retificador, as impedâncias vista pelo gerador piezoelétrico são apresentadas na Tabela 3 em conjunto com a potência gerada. Percebe-se que a impedância diminui com a elevação da frequência 
Tabela 2: Potência Instantânea Gerada $[m W]$ com Carga Puramente Resistiva em Função dos Modos de Vibração Naturais da Viga

\begin{tabular}{c|c|c|c|c}
\hline Resistência $[\Omega]$ & $1^{\circ}$ Modo & $2^{\circ}$ Modo & $3^{\circ}$ Modo & $4^{\circ}$ Modo \\
\hline \hline $1 k$ & 0.126 & 2.772 & 1.266 & 16.94 \\
\hline $5 k$ & 0.611 & 1.223 & 4.757 & 45.95 \\
\hline $10 k$ & 1.108 & 23.04 & 70.68 & 47.49 \\
\hline $20 k$ & 2.257 & 38.68 & 69.08 & 33.72 \\
\hline $50 k$ & 4.677 & 41.78 & 35.97 & 13.83 \\
\hline $100 k$ & 9.571 & 24.95 & 16.97 & 6.491 \\
\hline $200 k$ & 9.997 & 13.41 & 8.507 & 3.238 \\
\hline $500 k$ & 7.914 & 5.043 & 3.229 & 1.281 \\
\hline $1 M$ & 3.194 & 2.753 & 1.636 & 0.661 \\
\hline
\end{tabular}

para os mesmos valores de $R_{L}$ e $C_{L}$, regidos pela resposta em frequência de um circuito $R C$ paralelo. Outro item observado é que a potência gerada pelo elemento piezoelétrico não é linear. Isto se dá devido ao fato de em baixas frequências a tensão armazenada no capacitor $C_{L}$ ser menor em função da descarga no resistor $R_{L}$. Com o aumento da frequência, um número maior de ciclos de tensão/corrente são retificados, elevando a tensão armazenada na carga. A Tabela 3 também apresenta os valores de tensão na carga em função da resistência e modos de vibração. Para a resistência de $1 k \Omega$, a tensão no primeiro modo de vibração é quase zero, elevando a $5.82 \mathrm{~V}$ no quarto modo. Esse comportamento é seguido paras as resistências de $10 k \Omega$ e $100 k \Omega$. A resistência de $1 M \Omega$ pode ser considerada como carga infinita (circuito aberto), tanto que a tensão retificada não sofre alteração significativa.

Tabela 3: Impedância $Z_{e f}$ Vista Pelo Gerador Piezoelétrico $[k \Omega]$, Potência Gerada $P$ em Função da Frequência e Resistência $[m W]$ e Tensão Contínua na Carga $[V]$ (Capacitor $\left.C_{L}=10 \mu F\right)$

\begin{tabular}{c|c|c|c|c|c|c|c|c|c|c|c|c}
\hline \multirow{2}{*}{ Resistência $[\Omega]$} & \multicolumn{3}{|c|}{$1^{\circ}$ Modo Modo } & \multicolumn{3}{c|}{$3^{\circ}$ Modo } & \multicolumn{3}{c}{$4^{\text {o }}$ Modo } \\
\cline { 2 - 13 } & $Z_{\text {ef }}$ & $P$ & $V_{\text {ref }}$ & $Z_{\text {ef }}$ & $P$ & $V_{\text {ref }}$ & $Z_{\text {ef }}$ & $P$ & $V_{\text {ref }}$ & $Z_{\text {ef }}$ & $P$ & $V_{\text {ref }}$ \\
\hline \hline $1 k$ & 1.808 & 0.256 & 0.21 & 1.707 & 0.227 & 1.18 & 1.623 & 0.218 & 3.15 & 1.528 & 0.206 & 5.82 \\
\hline $10 k$ & 9.328 & 1.207 & 1.87 & 7.817 & 1.02 & 10.13 & 5.953 & 0.755 & 22.27 & 4.420 & 0.526 & 33.11 \\
\hline $100 k$ & 63.25 & 8.194 & 14.66 & 27.31 & 3.03 & 39.46 & 12.24 & 1.195 & 39.76 & 6.725 & 0.620 & 39.82 \\
\hline $1 M$ & 98.56 & 12.37 & 38.32 & 36.07 & 3.28 & 39.67 & 13.35 & 1.189 & 39.87 & 6.950 & 0.616 & 39.88 \\
\hline
\end{tabular}

\section{Conclusões}

Neste artigo, é feita a avaliação de um sistema de micro geração de energia baseado no efeito piezoelétrico submetido a diferentes tipos de cargas. Dois tipos de cargas (resistiva e não linear) foram investigados, sendo analisada a sua influência nas características do sistema e na potência gerada.

A carga puramente resistiva evidencia o comportamento de geração onde existe um ponto ótimo entre a frequência de excitação e a carga utilizada, bem como a influência da carga no modo de vibração do sistema. O comportamento do sistema com a inclusão 
do retificador de onda completa muda de forma expressiva, iniciando pela da tensão que é gerada na saída do elemento piezoelétrico, que tem característica diferente da excitação. A impedância vista pela carga varia com o modo de vibração, para resistência de $1 k \Omega$ a impedância cai aproximadamente $5 \%$ a cada modo, para $10 k \Omega$ cai $25 \%$, para $100 k \Omega$ em média $112 \%$ e para $1 M \Omega$ em média $145 \%$. A potência gerada é reduzida com a elevação dos modos de vibração, sendo que para resistência de $1 M \Omega$ a potência diminui $4 \mathrm{x}$ do $1^{\circ}$ para o $2^{\circ}$ modo de vibração, para resistência de $10 k \Omega$ a queda é de $15 \%$. Esta ocorrência está ligada ao fato que no $1^{\circ}$ modo de vibração ocorre a maior deformação na viga.

\section{Agradecimentos}

Agradecemos a CAPES pela concessão de apoio financeiro para o Mestrando Marcos Antonio Jeremias Coelho e a FAPERGS pelo apoio à pesquisa PqG 2110-2551/13-0.

\section{Referências}

[1] L. A. Aguirre, Introdução à Identificação de Sistemas - Técnicas Lineares e NãoLineares Aplicadas a Sistemas Reais, Editora UFMG, 4 a Edição, (2015).

[2] S.W. Arms, C.P. Townsend, D.L. Churchill, J.H. Galbreath e S.W. Mundell, Power management for energy harvesting wireless sensors, SPIE International Symposium on Smart Structures and Smart Materials, vol. 1, 1-9, (2005).

[3] S. P. Beeby, M. J. Tudor e N. M. White, Energy harvesting vibration sources for microsystems applications, Measurement Science and Technology, vol. 17, R175-R195, (2006), DOI: 10.1088/0957-0233/17/12/R017.

[4] D. Henrion, C. Prieur e S. Tliba, Improving conditioning of polynomial pole placement problems with application to low-order controller design for a flexible beam, LAASCNRS Research Report, vol. 1, 04163, (2004).

[5] J. Hu, J. Jong e C. Zhao, Vibration energy harvesting based on integrated piezoelectric components operating in different modes. IEEE Transactions on Ultrasonics, Ferroelectrics, and Frequency Control, vol. 57, 386-394, (2010), DOI: 10.1109/TUFFC.2010.1418.

[6] IEEE, IEEE standard on piezoelectricity, ANSI/IEEE Std 176-1987, 1-66, (1988).

[7] J. Liang e W. Liao, Impedance modeling and analysis for piezoelectric energy harvesting systems. IEEE/ASME Transactions on Mechatronics, vol. 17, 1145-1157, (2012), DOI: 10.1109/TMECH.2011.2160275.

[8] S.O. Reza Moheimani e A. J. Fleming, Piezoelectric Transducers for Vibration Control and Damping, Springer London, $1^{\mathrm{a}}$ Edição, (2006).

[9] S. Priya e D. J. Inman, Energy Harvesting Technologies, Springer, (2009). 